\title{
The Non-Uniform Grid in the FDTD Modeling of the Earthing Conductor in the Transient Grounding Resistance Analysis
}

\author{
Xu Jian-Hong, Mao Yun-Fei, Chen Jian \\ The China Satellite Maritime Tracking and Control Department, Yuan Wang III, Jiangyin 214431, China \\ xujianhong1111@163.com, maoyunfei4494@163.com, chenjian@163.com
}

\begin{abstract}
The grounding system plays an important part in the lightning protection system of power and communication systems. The finite-difference time-domain (FDTD) method is widely used in modeling complex electromagnetic interaction problems. However, it is difficult to model the earthing conductor using the standard FDTD method in the transient grounding resistance analysis, for the electrically small depth of the earthing conductors. In this work, nonuniform grid in the FDTD methods, which is typically used to resolve fine structures, is introduced into reduce the computational domain and therefore lead to a reduction of the computational cost. To further reduce the computational memory, the uniform grids are used in the electrode length direction while non-uniform grids are occupied in the electrode sectional directions. The efficiency of the proposed model has been approved by verifying both the electromagnetic field components near the earthing conductor and the grounding resistance.

Index Terms - Non-Uniform, FDTD Modeling, Earthing Conductor.
\end{abstract}

\section{I . Introduction}

Sensitive electronic components have been increasingly used lately both in power and communication systems. These components may suffer logic upset or damage at lower levels of induced electromagnetic interferences brought about by the lightning. As a result, evaluation of the transient grounding resistance of the grounding systems in the lightning protection systems has recently attracted considerable attentions [1]-[5].

The finite-difference time-domain (FDTD) method [6], which provides a simple and efficient way of solving Maxwell' equations for a variety of problems, has been widely applied in solving many types of electromagnetic problems. It is good at predicting the electromagnetic characteristics of a particular structure for it provides extensive time-domain information and the frequency-domain information can be provided via a discrete Fourier transform.

However, when the grounding resistance analysis of the lightning protection systems is involved, it will results in a huge memory and time usage with the uniform FDTD grids. Because the dimension of the grounding electrode and the reference electrode are so small compared with the total computational domain, and one has to refine the FDTD grid to the conductor dimension, which will result in huge memory usage.

Several papers have introduced different methods to reduce the truncation error at grid boundary. In [7], two methods were introduced to maintain second-order accuracy. One method uses an appropriate mesh ratio between two regions to obtain the central finite differences. The other method uses a universal grading scheme with continuously variable lattice size; but a demonstration of the effectiveness of this method was not reported. The non-uniform grid technology in the FDTD method, which is typically used to resolve fine structures, can reduce the computational domain and therefore lead to a reduction of the computational cost [8]. Considering electrically small size of the grounding electrode in the sectional area dimensions, the non-uniform grid is introduced into the FDTD method in two dimensions to deal with the electrode areas in the grounding resistance analysis of the lightning protection systems. The efficiency of the proposed model has been approved by verifying both the field distribution near the earthing conductor and the grounding resistance.

\section{II . The Grounding Resistance Calculation Model}

To calculate the grounding resistance, a grounding model is adopted as shown in Fig.1, where earth is used as the return path [9-11]. It is assumed that constitutive parameters of the ground are constant, and the relative permittivity of the ground is set as 9.0 and the conductivity is $0.001 \mathrm{~S} / \mathrm{m}$. To be simply, a single earthing conductor is used, and a remote electrode is used to permit the transient current flowing into the earth.

The grounding resistance is defined as a ratio of the transient voltage to the transient current

$$
R_{t}=V_{t} / I_{t}
$$

Here $I_{t}$ is the transient current flowing through grounding conductor, which can be defined from the Ampere's Law

$$
\begin{aligned}
I_{t}= & {\left[H_{z}\left(i-\frac{1}{2}, j+\frac{1}{2}, k\right)-H_{z}\left(i+\frac{1}{2}, j+\frac{1}{2}, k\right)\right] \Delta z } \\
& +\left[H_{x}\left(i, j+\frac{1}{2}, k+\frac{1}{2}\right)-H_{x}\left(i, j+\frac{1}{2}, k-\frac{1}{2}\right)\right] \Delta x
\end{aligned}
$$

In the FDTD analysis, the voltage between the two sides of a cell can be defined as

$$
V_{j}=-E_{j} \cdot \Delta s_{j}
$$




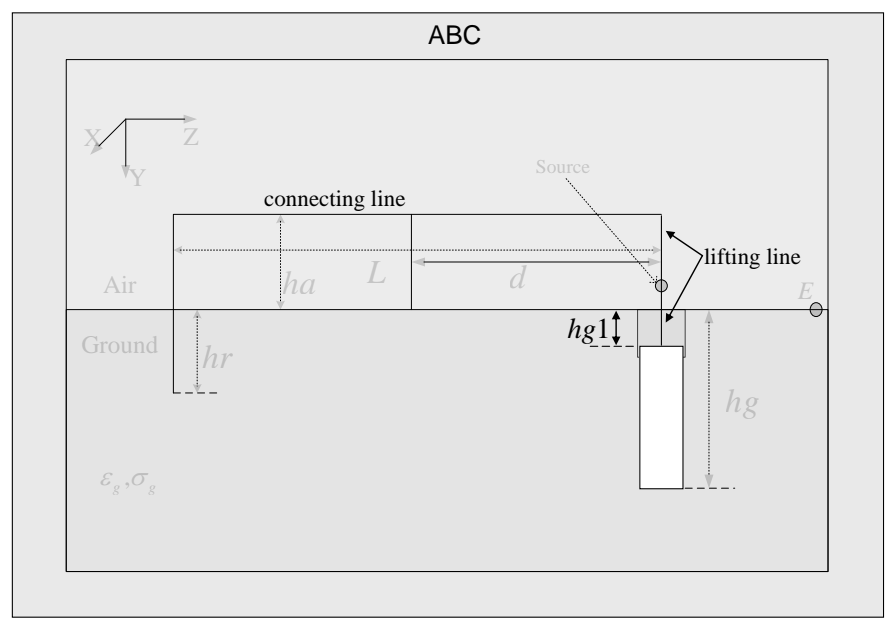

Fig.1.The grounding system resistance analysis model.

By integrating the electric field along the air-ground interface from the computational domain boundary (line $E$ of Fig.1) to the lifting line, the transient voltage can be obtained

$$
V_{t}=\sum_{j=N_{l}}^{N_{e}} V_{j}=-\sum_{j=N_{l}}^{N_{e}} E_{j} \Delta s_{j}
$$

where $N_{l}$ and $N_{e}$ are the FDTD mesh index of the point where the lifting line entering underground and the point $E$ of Fig.1 respectively.

The double-exponential lightning pulse is applied as the voltage source

$$
V=k V_{0}\left(e^{-\alpha t}-e^{-\beta t}\right)
$$

where $\alpha=3.7618 \times 10^{4} \mathrm{~s}^{-1}, \beta=1.13643 \times 10^{7} \mathrm{~s}^{-1}, k=1.02, V_{0}=5.4 \mathrm{kV}$. The computing domain is terminated by 10-layer the convolution PML [12-13].

\section{The Non-Uniform FDTD Algorithm}

The grounding electrodes are always several meters in length while electrically small in the sectional area dimensions [1]. To model the grounding electrode accurately without result in huge computational resources, the non-uniform FDTD method is involved [8]. To save computational resources, nonuniform grids in the $x$ - and $z$-direction are occupied while uniform grid in the $y$-direction is used.

The adjacent non-uniform grids are as shown in Fig.2, where $k$ is the expansion factor. It can be seen that the magnetic the magnetic field components are still located at the center of the FDTD grids, while the electric field components are no longer at the center of the grids, thus the non-uniform scheme is no a second-order method at all. However, the accuracy can be improved by limit the expansion factor to a size.

For the no-uniform grids areas, the time-stepping equations for the electric field components can be written as

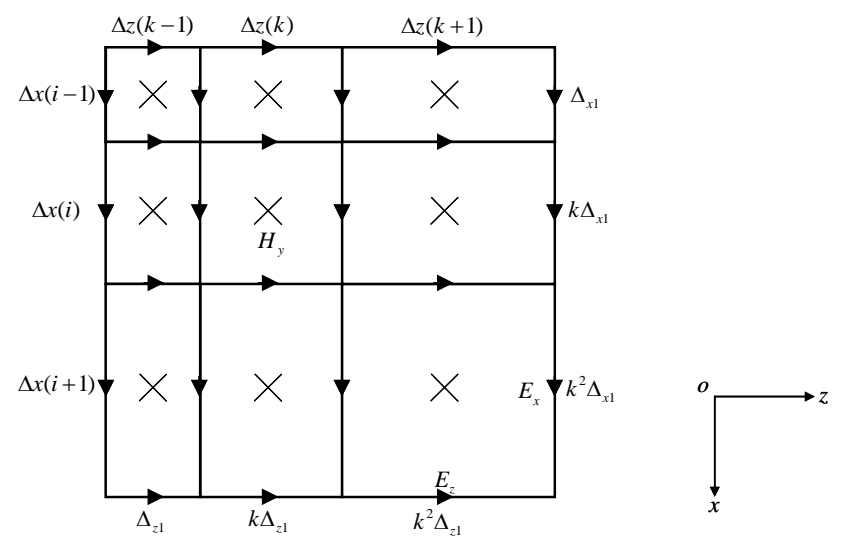

Fig. 2. The cross section of angle iron, where $\mathrm{b} 1=\mathrm{b} 2=5 \mathrm{~cm}, \mathrm{~h}=0.5 \mathrm{~cm}$

$$
\begin{array}{r}
E_{x}^{n+1}\left(i+\frac{1}{2}, j, k\right)=\mathrm{CA} \square E_{x}^{n}\left(i+\frac{1}{2}, j, k\right) \\
+C B\left[\frac{H_{z}^{n+1 / 2}\left(i+\frac{1}{2}, j+\frac{1}{2}, k\right)-H_{z}^{n+1 / 2}\left(i+\frac{1}{2}, j-\frac{1}{2}, k\right)}{\Delta y(j)}\right. \\
\left.-\frac{H_{y}^{n+1 / 2}\left(i, j+\frac{1}{2}, k+\frac{1}{2}\right)-H_{y}^{n+1 / 2}\left(i, j+\frac{1}{2}, k-\frac{1}{2}\right)}{0.125(\Delta z(k-1)+6 \Delta z(k)+\Delta z(k+1))}\right] \\
E_{y}^{n+1}\left(i, j+\frac{1}{2}, k\right)=\mathrm{CA}\left[E_{y}^{n}\left(i, j+\frac{1}{2}, k\right)\right. \\
+C B \square\left[\frac{H_{x}^{n+1 / 2}\left(i, j+\frac{1}{2}, k+\frac{1}{2}\right)-H_{x}^{n+1 / 2}\left(i, j+\frac{1}{2}, k-\frac{1}{2}\right)}{0.125(\Delta z(k-1)+6 \Delta z(k)+\Delta z(k+1))}\right. \\
\left.-\frac{H_{z}^{n+1 / 2}\left(i+\frac{1}{2}, j+\frac{1}{2}, k\right)-H_{z}^{n+1 / 2}\left(i-\frac{1}{2}, j+\frac{1}{2}, k\right)}{0.125(\Delta x(i-1)+6 \Delta x(i)+\Delta x(i+1))}\right]
\end{array}
$$

$$
\begin{aligned}
E_{z}^{n+1}\left(i, j, k+\frac{1}{2}\right) & =\mathrm{CA} \sqsubset E_{z}^{n}\left(i, j, k+\frac{1}{2}\right) \\
+ & C B\left[\frac{H_{y}^{n+1 / 2}\left(i+\frac{1}{2}, j, k+\frac{1}{2}\right)-H_{y}^{n+1 / 2}\left(i-\frac{1}{2}, j, k+\frac{1}{2}\right)}{0.125(\Delta x(i-1)+6 \Delta x(i)+\Delta x(i+1))}\right. \\
& \left.-\frac{H_{x}^{n+1 / 2}\left(i, j+\frac{1}{2}, k+\frac{1}{2}\right)-H_{x}^{n+1 / 2}\left(i, j-\frac{1}{2}, k+\frac{1}{2}\right)}{\Delta y(j)}\right]
\end{aligned}
$$

In (6) to (8), the coefficients $C A$ and $C B$ are obtained from

$$
\begin{aligned}
& C A=\frac{\varepsilon-0.5 \Delta t \sigma}{\varepsilon+0.5 \Delta t \sigma} \\
& C B=\frac{\Delta t}{\varepsilon+0.5 \Delta t \sigma}
\end{aligned}
$$

where $\varepsilon$ is the permittivity and $\sigma$ is the conductivity.

The time marching equation for the magnetic field components in the non-uniform areas can be written as

$$
\begin{aligned}
H_{x}^{n+1 / 2}\left(i, j+\frac{1}{2}, k+\frac{1}{2}\right)= & C P \square H_{x}^{n-1 / 2}\left(i, j+\frac{1}{2}, k+\frac{1}{2}\right) \\
& +C Q \llbracket\left[\frac{E_{z}^{n}\left(i, j+1, k+\frac{1}{2}\right)-E_{z}^{n}\left(i, j, k+\frac{1}{2}\right)}{\Delta y(j)}\right. \\
& \left.-\frac{E_{y}^{n}\left(i, j+\frac{1}{2}, k+1\right)-E_{y}^{n}\left(i, j+\frac{1}{2}, k\right)}{0.125(\Delta z(k-1)+6 \Delta z(k)+\Delta z(k+1))}\right]
\end{aligned}
$$




$$
\begin{aligned}
H_{y}^{n+1 / 2}\left(i+\frac{1}{2}, j, k+\frac{1}{2}\right)=C P \llbracket H_{y}^{n-1 / 2}\left(i+\frac{1}{2}, j, k+\frac{1}{2}\right) \\
+C Q\left[\frac{E_{x}^{n}\left(i+\frac{1}{2}, j, k+1\right)-E_{x}^{n}\left(i+\frac{1}{2}, j, k\right)}{0.125(\Delta z(k-1)+6 \Delta z(k)+\Delta z(k+1))}\right. \\
\left.-\frac{E_{z}^{n}\left(i+1, j, k+\frac{1}{2}\right)-E_{z}^{n}\left(i, j, k+\frac{1}{2}\right)}{0.125(\Delta x(i-1)+6 \Delta x(i)+\Delta x(i+1))}\right] \\
H_{z}^{n+1 / 2}\left(i+\frac{1}{2}, j+\frac{1}{2}, k\right)=C P \square H_{z}^{n-1 / 2}\left(i+\frac{1}{2}, j+\frac{1}{2}, k\right) \\
+C Q\left[\frac{E_{y}^{n}\left(i+1, j+\frac{1}{2}, k\right)-E_{y}^{n}\left(i, j+\frac{1}{2}, k\right)}{0.125(\Delta x(i-1)+6 \Delta x(i)+\Delta x(i+1))}\right. \\
\left.-\frac{E_{x}^{n}\left(i+\frac{1}{2}, j+1, k\right)-E_{x}^{n}\left(i+\frac{1}{2}, j, k\right)}{\Delta y(j)}\right]
\end{aligned}
$$

In (11) to (13), the coefficients $C P$ and $C Q$ are obtained from

$$
\begin{gathered}
C P=\frac{\mu-0.5 \Delta t \sigma_{m}}{\mu+0.5 \Delta t \sigma_{m}} \\
C Q=\frac{\mu-0.5 \Delta t \sigma_{m}}{\mu+0.5 \Delta t \sigma_{m}}
\end{gathered}
$$

where $\varepsilon$ is the permittivity and $\sigma$ is the conductivity.

It worth to note that the expansion factor of the nearby grids should not be so large and it is needed

$$
1.2 \Delta_{x}(i-1) \leq \Delta_{x}(i) \leq \Delta_{x}(i+1) / 1.2
$$

Additionally, the dimension of the largest grid can not be lager than 20 times of the smallest ones.

The time step is limited by the Courant-Friedrichs-Lewy

$$
\Delta t \leq \frac{1}{\sqrt{\frac{1}{\left(\Delta x_{\min }\right)^{2}}+\frac{1}{\left(\Delta y_{\min }\right)^{2}}+\frac{1}{\left(\Delta z_{\min }\right)^{2}}}}
$$

where $\Delta x_{\min }, \Delta y_{\min }$ and $\Delta z_{\min }$ are the smallest grid dimensions in the non-uniform grids area.

With this model, the sectional areas of the earthing electrodes can be modeled with fine FDTD grids without result in huge computational resources.

\section{Numerical Results}

To validate the efficiency of proposed method, the standard FDTD simulation result is included as a benchmark for comparison. It is agreed that a standard FDTD simulation with a sufficient slot resolution will be sufficiently accurate to be considered as a reference.

The computational model shown Fig.1 is adopted. We set the parameters as $L=9 \mathrm{~m}, h g 1=0.3 \mathrm{~m}, h g=1.8 \mathrm{~m}, h r=2.5 \mathrm{~m}$, $h a=0.8 \mathrm{~m}$. The lifting line, connecting line and remote current electrode are all round steel with a diameter of $0.025 \mathrm{~m}$. The computing domain, which is $x \times y \times z=4.0 \mathrm{~m} \times 4.6 \mathrm{~m} \times 11.0 \mathrm{~m}$, is terminated by the convolution PML [10].

The grid size of the reference simulation is $\Delta=1 \mathrm{~cm}$, and the time step is $\Delta t=\Delta / 2 c$, where $c$ is the speed of the light in the free space.
The uniform grid size is $\Delta_{x} \times \Delta_{y} \times \Delta_{z}=10 \mathrm{~cm} \times 10 \mathrm{~cm} \times 10 \mathrm{~cm}$ for the main areas. The expansion factor is set as $k=k_{x}=k_{z}=1.162$, while uniform grid is used in the $y$ direction, as shown in Fig.1 (b). 20 layers non-uniform grid is used, which results in the smallest cell size $\Delta_{\min }=\Delta x_{\min }=\Delta z_{\min }=0.5 \mathrm{~cm}$ for the electrode areas, and the time step is $\Delta t=\Delta_{\min } / 2 c$.

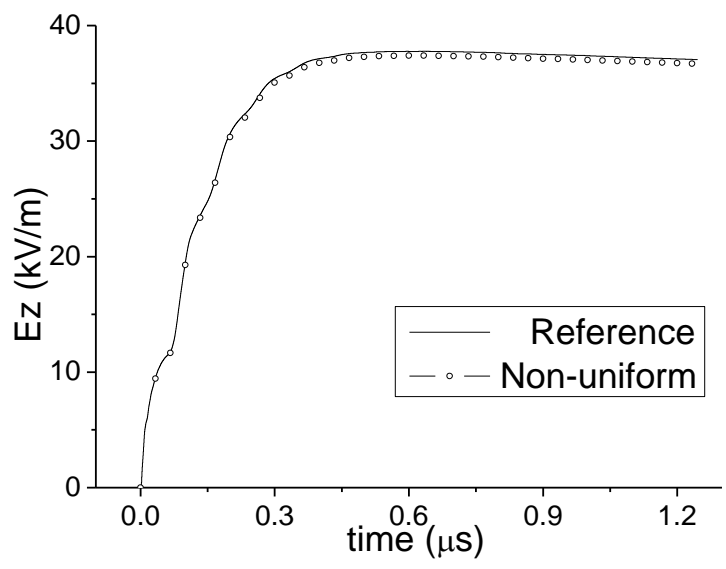

Fig.3. The $E_{z}$ variation in the $\mathrm{z}$ direction near the angle iron.

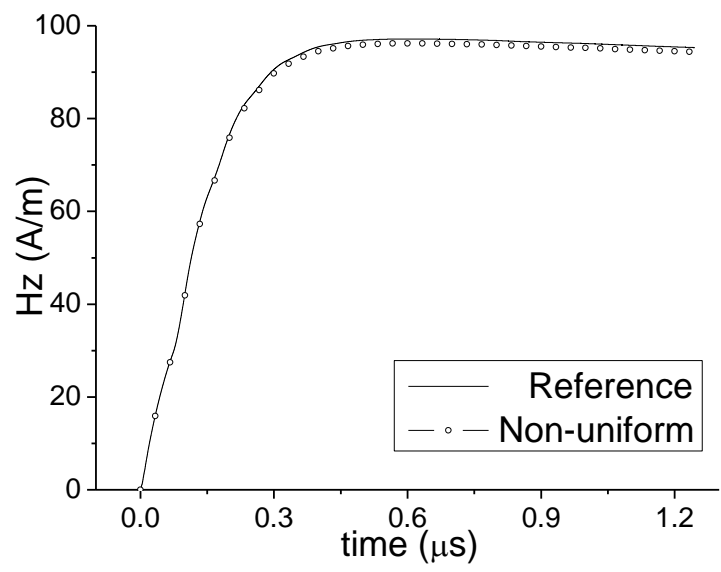

Fig.4. The $E_{z}$ variation in the $\mathrm{z}$ direction near the angle iron.

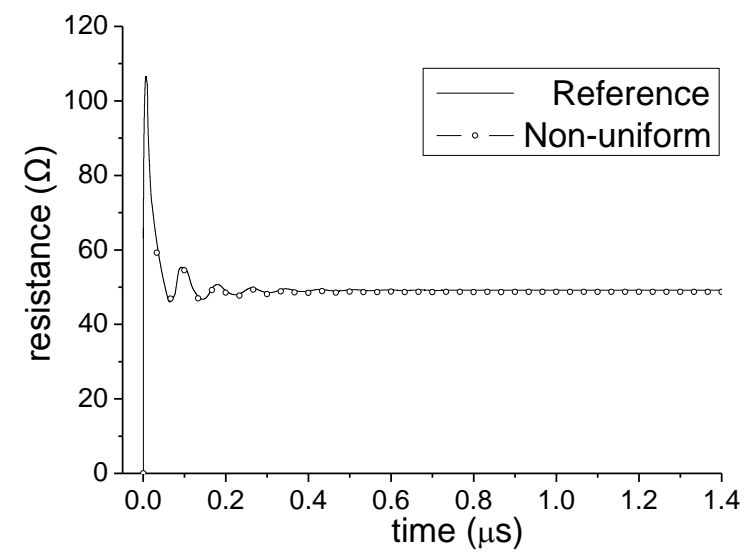

Fig.5. The validation of the proposed method for fine cross section simulation. 


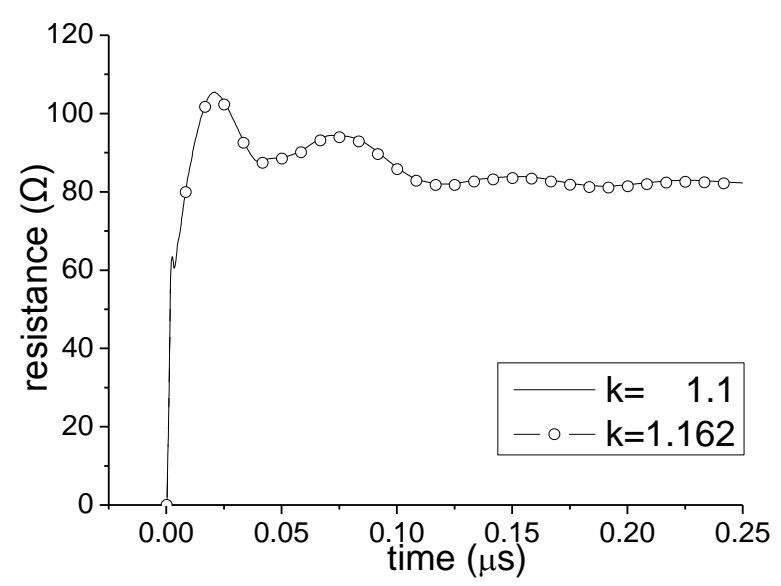

Fig.6. The $E_{z}$ variation in the $\mathrm{z}$ direction near the angle iron.

Firstly, a $6 \mathrm{~cm} \times 6 \mathrm{~cm} \times 250 \mathrm{~cm}$ earthing conductor is involved. The electric and magnetic field components $2 \mathrm{~cm}$ away from the conductor are monitored, as shown in Fig.3 and Fig. 4 respectively. It can be seen that the proposed method simulates the electromagnetic filed components accurately.

The resistance of the grounding system is also calculated, as graphed in Fig.5. It can be seen that the proposed model is an accurate modeling of the grounding system resistance.

Secondly, we also tested the proposed method performance when the expansion factor $k$ the grounding resistance of another $1.5 \mathrm{~m}$ long angle iron. The expansion factor is $k=1.1$ and $k=1.162$ respectively, and the grounding resistance is plotted in Fig.6. It can be seen that the result given by the two conditions are in good agreement with each other.

Form the validation of the electromagnetic field components and the grounding resistance of two earthing conductors, the efficiency of the proposed method is validated. However, the memory usage of the proposed method is $52 \mathrm{MB}$, while the standard FDTD simulation occupied 1.8 GB memory.

\section{Conclusion}

In this work, non-uniform grid in the FDTD methods, which is typically used to resolve fine structures, is introduced into reduce the computational domain and therefore lead to a reduction of the computational cost. To further reduce the computational memory, the uniform grids are used in the electrode length direction while non-uniform grids are occupied in the electrode sectional directions.

The accuracy of the proposed method has been approved from the validation of the electromagnetic field components and the grounding resistance result. It is demonstrated that the much computational memory is saved when the proposed method is used compared with the standard fine grid FDTD simulation.

With the proposed method, the earthing conductor of the grounding systems in the grounding resistance analysis can be modeled without involving huge computational resources.

\section{References}

[1] IEC 62305-3, Ed. 1, "Protection against lightning - Part 3: Physical damage to structures and life hazard," 2004.

[2] S. Visacro and R. Alipio, "Frequency dependence of soil parameters: Experimental results, predicting formula and influence on the lightning response of grounding electrodes," IEEE Trans. on Power Delivery, Vol. 27, No. 2, 927\{935, 2012.

[3] R. Zeng, J. L. He, Y. Q. Gao, J. Zou, and Z. C. Guan, "Grounding resistance measurement analysis of grounding system in vertical-layered soil," IEEE Trans. on Power Delivery, Vol. 19, No. 4, 1553-1559, 2004.

[4] A. P. S. Meliopoulos, S. Patel, and G. J. Cokkinides, "A new ethod and instrument for touch and step voltage measurement," IEEE Trans. on Power Delivery, Vol. 9, No. 4, 1850-1860, Oct. 1994.

[5] M., Y. Baba Tsumura, N. Nagaoka, and A. Ametani, "FDTD simulation of a horizontal grounding electrode and modeling of its equivalent circuit," IEEE Trans. on Electromagnetic Compatibility, Vol. 48, No. 4, 817-825, 2006.

[6] Taflove and Hagness, Computational Electrodynamics: The FiniteDifference Time-Domain Method, Artech House, 3rd ed., 2005.

[7] S. Xiao and R. Vahldieck, "An improved 2D-FDTDalgorithmfor hybrid mode analysis of quasi-planar transmission lines," in IEEE MTT-S Tech. Dig., vol. 1, 1993, pp. 421-424.

[8] W. Yu and R. Mittra, "A technique of improving the accuracy of the non-uniform time-domain algorithm", IEEE Transactions on MTT, Vol.47, No.3, 353-356, 1999.

[9] R. Xiong, B. Chen, Y.-F. Mao, W. Deng, and Q. Wu, "FDTD modeling of the earthing conductor in the transient grounding resistance analysis," IEEE Antennas and Wireless Propagat. Lett., Vol. 11, 957-960, 2012.

[10] R. Xiong, B. Chen, J.-J. Han, Y.-Y. Qiu, W. Yang, and Q. Ning, "Transient resistance analysis of large grounding systems using the FDTD method," Progress In Electromagnetic Research, Vol.132, 159175, 2012.

[11] R. Xiong, B. Chen, L-H. Shi, Y-T. Duan and G. Zhang, "An efficient method to reduce the peak transient grounding resistance value of a grounding system," Progress In Electromagnetic Research, Vol.138, $255-267,2013$.

[12] J. A. Roden and S. D. Gedney, "Convolution PML (CPML): An efficient FDTD implementation of the CFS-PML for arbitrary media", Microwave and Optical Technology Letters, Vol.27, No.5, pp. 334-339, Dec.2000.

[13] Mao, Y.-F., B. Chen, H.-Q. Liu, J.-L. Xia, and J.-Z. Tang, "A hybrid implicit-explicit spectral FDTD scheme for the oblique incidence programs on periodic structures," Progress In Electromagnetics Research, Vol. 128, 153-170, 2012. 